\title{
Gender and visuospatial processing in multimedia STEM learning
}
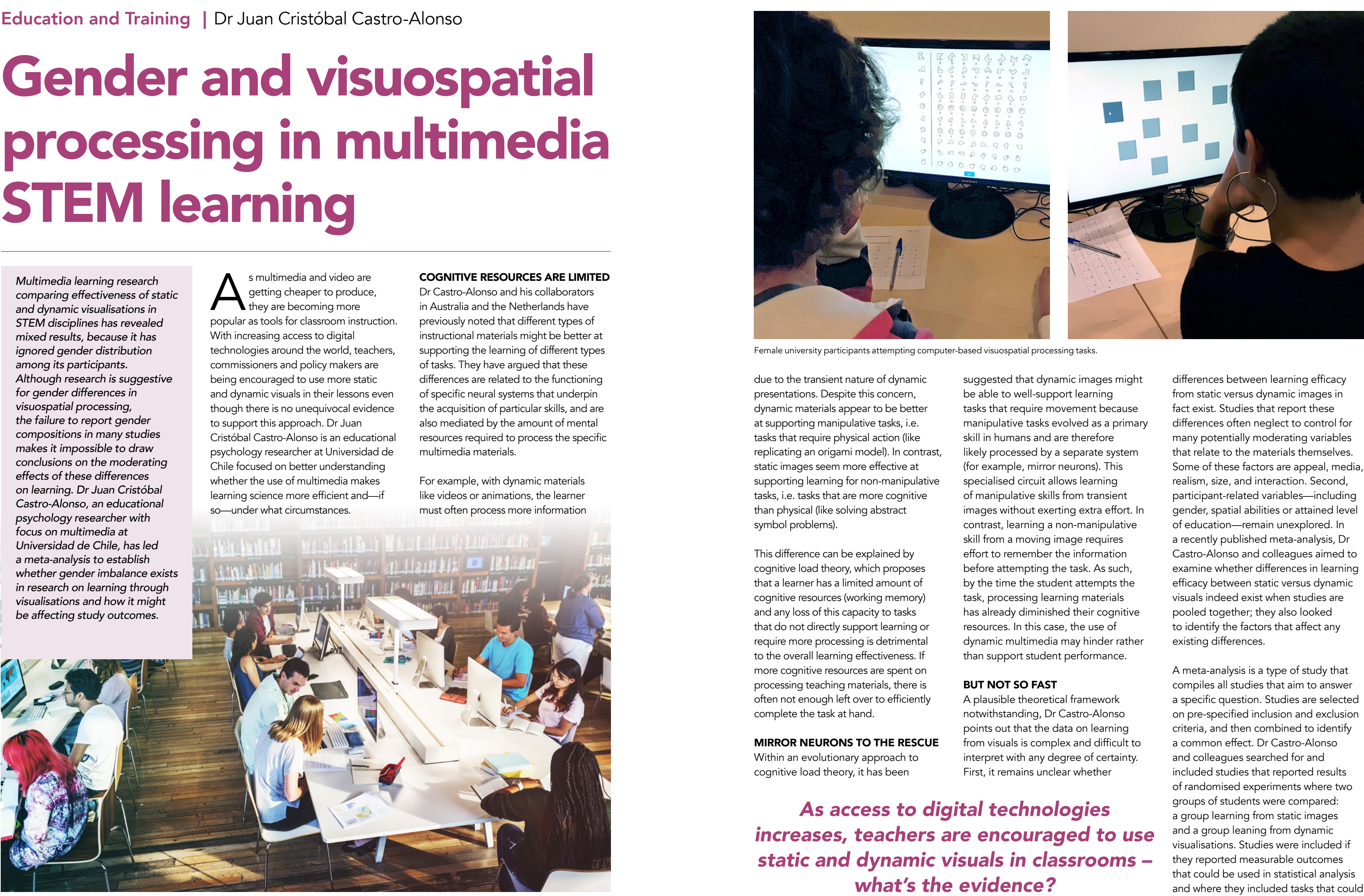

Female university participants attempting computer-based visospatia processing tasks

due to the transient nature of dynamic presentation dynamic materials appear to be better at supporting manipulative tasks, i.e. tasks that require physical action (like replicating an origami model). In contrast, static images seem more effective at supporting learning for non-manipulative tasks, i.e. tasks that are more cognitive than physical (like solving abstract symbol problems).

This difference can be explained by cognitive load theory, which proposes that a liver has al which prount of cognitive resources (working memory) that do not directly support learning or require more processing is detrimenta to the overall learning effectiveness. If more cognitive resources are spent on processing teaching materials, there is often not enough left over to efficiently complete the task at hand.

MIRROR NEURONS TO THE RESCUE Within an evolutionary approach to cognitive load theory, it has been

As access to digital technologies increases, teachers are encouraged to use static and dynamic visuals in classrooms what's the evidence? differences between learning efficacy (ngested that dynic images might be able to well-support learning manipulative tasks evolved as a prise skill in humans and are therefore likely processed by a separate system (for example, mirror neurons). This specialised circuit allows learning images without exerting extra effort. contrast, learning a non-manipulative skill from a moving image requires effort to remember the information by the time the student attempts the task, processing learning materials has already diminished their cognitive resources. In this case, the use of dynac multimedia may hinder rather than support student performance.

BUT NOT SO FAST

A plausible theoretical framework notwithstanding, Dr Castro-Alonso
points out that the data on learning from visuals is complex and difficult to interpret with any degree of certain
First, it remains unclear whether from static versus dynamic images in fact exist. Studies that report these differences often neglect to control for many potentially moderating variables that relate to the materials themselves. Some of these factors are appeal, media, realism, size, and interaction. Second, participant-related variables-including gender, spatial ablities or attained leve a education remain unexplored. In Castrapso examine whothd colleagues aimed to efficacy between static versus dynamic visuals indeed exist when studies are pooled together; they also looked identify the factors that affect any existing differences.

A meta-analysis is a type of study that compiles all studies that aim to answer a specific question. Studies are selected on pre-specified inclusion and exclusio criteria, and then combined to identify a common effect. Dr Castro-Alonso included studies that reported results

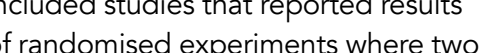
groups of students were compared: group learning from static images and a group leaning from dynamic vsualisations. Studies were included if they reported measurable outcomes and where they included tasks thalysis 


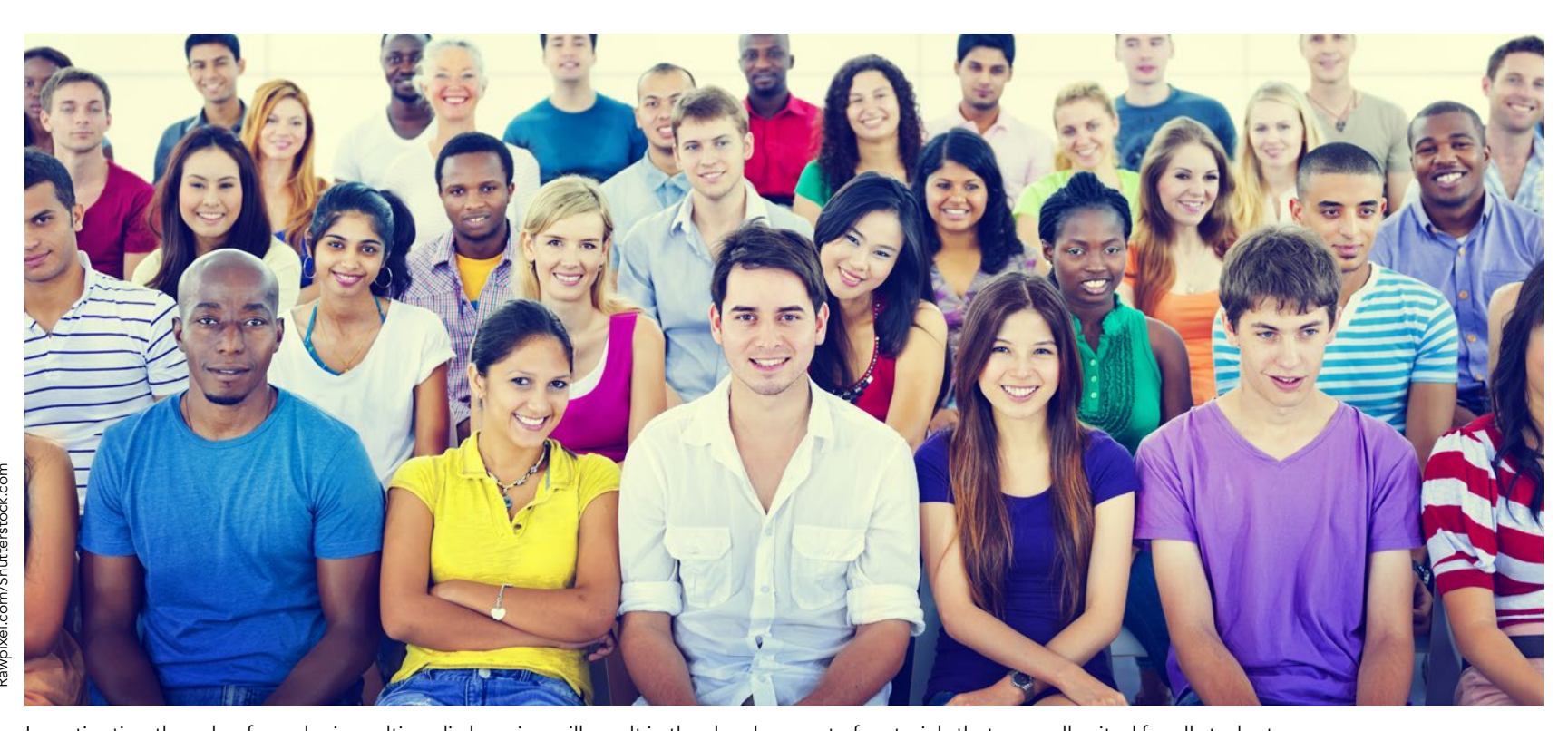

be categorised as either STEM-related (subjects including science, technolog manipulative-procedural (such as folding). Studies were excluded if the gender ratio for a participant sample was not reported. Their findings have been recently published in Educational Psychology Review.

\section{IN SEARCH OF CLARITY}

After combining and analysing results from 46 studies that included over

5,000 participants, Dr Castro-Alonso and colleagues reported that there was indeed some difference in the efficacy of learning in the groups exposed to the static versus dynamic images. Students that worked with dynamic visuals performed slightly better. Ge visualisations in samples that included more females, static visualisations performed better; samples with few females and more males seemed to favour dynamic visualisations.

At the same time, the researchers reported that the level of education attained by study participants, as well as he type of media involved in the study, also affected how participants learned from different visualisations.

\section{MEN AND WOMEN LEARN} DIFFERENTLY...PROBABLY vidence that gender affects multimedia-based learning is the key insight from this study. It is especialy
Researchers so far have sometimes failed to report whether studies have been done on males or females.

exploring possible differences in multimedia learning between males and females is scarce. In research concerning the use of visuals in the classroom, gender of study participants is often neglected as a possible moderator, so mach so that relevant ratios are often left out of study reports. In Dr CastroAlonso's study, 48 of all eligible studies had to be excluded because they didn't report gender ratios. This is an important issue for at least two reasons. First, not designs and in data an as not reporting genderritios, has produced a body of research that is unclear and difficult to interpret. Second these findings are often used as basis for policy recommendations, even though they lack a strong evidence base.

\section{LOOKING FORWARD}

Building on the results of their metaa collaboration that aims to better understand the role of gender in learning science from multimedia materials. Specifically, he and colleagues will examine how cognitive load is moderated by students' characteristic in immediate and delayed tests in learning about rotation and trans
of organic chemistry molecular gender differences in STEM education analysis, Dr Castro-Alonso is leading representations-which is often challenging for undergraduates.

The study is designed to involve a randomised sample of 720 undergraduates in Chile (50\% female) and is combined with outreach seminars to increase teachers 'and students' awareness about cognitive load and Once the study has been completed, scientifc commanity through publications

Dr Castro-Alonso hopes that greater insight into the role of gender in learning science and other STEM topics through multimedia will result in the development of study materials that are well suited for all students. In addition, he also hopes that engagement with the public will increase awareness of the evidence behind teaching and learning recommendations and will result in policies and investments that are supported by scientific methods. A highly recommended resource to gain such insights is Dr Castro-Alonso's book on Visuospatial processing for education by Springer Ltod, where leading world
experts discuss a number of critical topics. the results will be shared with the global in health and natural sciences' (published

\section{Research Objectives}

Dr Juan C. Castro-Alonso's research uses quantitative randomised controlled experiments to investigate the variables influencing the teaching and learning of STEM topics, with particular focus on integrative teaching methods, including multimedia.

\section{Detail}

Dr Juan Cristóbal Castro-Alonso

Periodista José Carrasco Tapia 75

Santiago,

Santiago (Chile), 833001

Dr Juan C. Castro-Alonso is Associate Researcher at the Center for Advanced Research in Education, Universidad de Chile. He is a Biochemist, Master in Communication and Education, and PhD in Education. He is academic editor of PLOS ONE and has coauthored more than 15 journal articles and book chapters.

Funding

PA Ex Eellence Project FBOOO3

CONICYT Fondecyt 11180255

\section{Collaborators}

(Pmeritus Professor Paul Ayres (p. ayres@unsw.edu.au). School of Education, University of New South Wales,

- Professor Fred Paas (paas@essb.eur.nl). Department of Psychology, Education, and Child Studies, Erasmus University Rotterdam, Rotterdam, The Netherlands. Early Start/School of Education, University of Wollongong, Wollongong, Australia.

- Dr Mona Wong (wpsmona@hku.hk). Faculty of Education The University of Hong Kong, Hong Kong, Hong Kong. Professor Olusola O. Adesope (olusola.adesope@wsu. edu). College of Education, Washington State University, Pullman, WA, USA

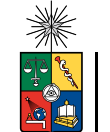

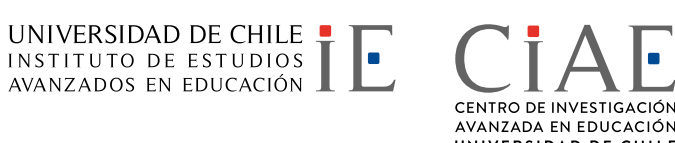

\section{References}

Castro-Alonso JC, Wong M, Adesope OO, Ayres P, Paas (2019). Gender imbalance in instructional dynamic versus static visualizations: A meta-analysis. Educational Psychology Review. 10.1007/s10648-019-09469-1

Castro-Alonso JC, Ayres P, Wong M, Paas F. (2018). Learning symbols from permanent and transient visual presentations 1-13. 10.1016/j.compedu.2017.08.011

Castro-Alonso JC, Ayres P, Paas F. (2016). Comparing apples and oranges? A critical look at research on learning from 234-243. 10.1016/j.compedu 2016.09.004

Castro-Alonso JC, Ayres P, Paas F. (2015). Animations showing Lego manipulative tasks: Three potential moderators of effectiveness. Computers and Education. 85: 1-13. 10.1016/j.compedu.2014.12.022

\section{Personal Response}

Gender is a variable normally reported in health and psychology research as one of the main moderators
of outcomes. Why do you think colleagues in your field have reluctantly reported on effects of gender in their studies? Do you think this is more or less likely to

II We don't know why gender is not being considered an important variable in educational multimedia research, or at least important enough to be reported in the investigated but we don't know the causes. I have not investigated the political climate, so I'm not sure if the climate is currently trying to equate women and men or acknowledging tha there are gender and sexual differences, including the differences that can influence visuospatial processing
and STEM multimedia learning. 